\title{
Image Analysis Determination of the Influence of Surface Structure of Silicone Rubbers on Biofouling
}

\author{
Sevil Atarijabarzadeh, ${ }^{1}$ Fritjof Nilsson, ${ }^{1,2}$ Henrik Hillborg, ${ }^{1,2}$ \\ Sigbritt Karlsson, ${ }^{1,3}$ and Emma Strömberg ${ }^{1}$ \\ ${ }^{1}$ KTH Royal Institute of Technology, School of Chemical Science and Engineering, Fibre and Polymer Technology, \\ Teknikringen 56-58, 10044 Stockholm, Sweden \\ ${ }^{2}$ ABB, Corporate Research, 72178 Västerås, Sweden \\ ${ }^{3}$ University of Skövde, P.O. Box 408, 54128 Skövde, Sweden
}

Correspondence should be addressed to Emma Strömberg; emmast@kth.se

Received 7 April 2015; Accepted 13 May 2015

Academic Editor: Christopher Batich

Copyright (C) 2015 Sevil Atarijabarzadeh et al. This is an open access article distributed under the Creative Commons Attribution License, which permits unrestricted use, distribution, and reproduction in any medium, provided the original work is properly cited.

\begin{abstract}
This study focuses on how the texture of the silicone rubber material affects the distribution of microbial growth on the surface of materials used for high voltage insulation. The analysis of surface wetting properties showed that the textured surfaces provide higher receding contact angles and therefore lower contact angle hysteresis. The textured surfaces decrease the risk for dry band formation and thus preserve the electrical properties of the material due to a more homogeneous distribution of water on the surface, which, however, promotes the formation of more extensive biofilms. The samples were inoculated with fungal suspension and incubated in a microenvironment chamber simulating authentic conditions in the field. The extent and distribution of microbial growth on the textured and plane surface samples representing the different parts of the insulator housing that is shank and shed were determined by visual inspection and image analysis methods. The results showed that the microbial growth was evenly distributed on the surface of the textured samples but restricted to limited areas on the plane samples. More intensive microbial growth was determined on the textured samples representing sheds. It would therefore be preferable to use the textured surface silicone rubber for the shank of the insulator.
\end{abstract}

\section{Introduction}

Silicone rubber (SIR) is extensively used as housing material for high voltage (HV) insulators. Low surface energy, high hydrophobicity, and good insulating properties make SIR a good candidate for housing material $[1,2]$. Severe pollution, heavy rain, and salt fog can result in a temporary loss of hydrophobicity of the SIR, but during longer dry periods and with cleaning the hydrophobicity is restored [3]. Surface biofouling as a result of microbial deposition on HV insulators and biofilm formation on the surface may also lead to a temporary loss of hydrophobicity [4-6]. Several studies have been conducted on the microbial contamination of traditional and modern HV insulators [5-12]. Gorur et al. [9] concluded that the microbial growth on nonceramic insulators depends on the formulation as well as on the environment. Visible mold growth was observed on one type of
SIR insulator located inland in the tropical environment of Florida. However, there was no visible growth on the same types of SIR insulator located along the coast or on insulators made of ethylene propylene rubber (EPR) or other types of SIR [9]. Wallström et al. developed several test methods to evaluate the biofilm contamination of the SIR insulators, for example, a rapid test of SIR resistance to molds [5]. In other studies by Wallström et al. [7, 8], microenvironment chambers simulating outdoor environments were designed to evaluate the extent of microbial growth on SIR materials containing different flame retardants under authentic conditions. Image analysis methods were developed to determine the growth rate [8] and to differentiate between clean and biocontaminated surfaces [13]. One of the methods focused on the study of microbial growth from a greater distance, which is necessary considering the position of the insulators in the field [14]. Inhibition of microbial growth, that is, preventing 


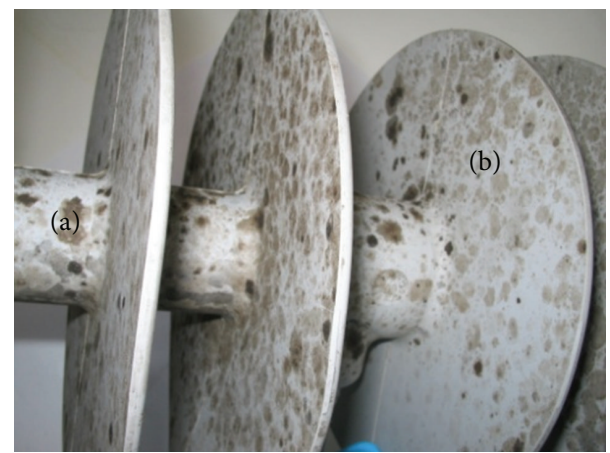

FIGURE 1: A SIR HV insulator covered by biofilm in (a) shank and (b) shed regions.

the formation of the biofilm, is desired to increase the life time of the HV insulators. As shown by Atarijabarzadeh et al. [15], the inhibition of microorganism growth can be achieved by the addition of suitable antimicrobial agents to SIR materials.

Loss of surface hydrophobicity and the formation of a film of water on the surface of the $\mathrm{HV}$ insulator result in leakage currents and discharges which may cause flashover. Dry bands are also formed due to evaporation of the water in some areas with a higher temperature. The dry bands may lead to electrical discharges which cause erosion of the polymeric insulators. As shown in Figure 1, the microbial growth is extensive on all surface regions of the insulators. The shank region of an insulator cannot be washed effectively, and pollution and microorganisms may accumulate. The density of the surface discharge current flowing between two ends of the insulator is higher in the shank region due to the geometry of the insulator. Consequently, the surface electric field strength is highest in this region, resulting in a greater risk of surface heating and hence thermal damage as a result of the formation of dry bands and partial arcs $[16,17]$.

Textured SIR surfaces have been designed and patented to improve the antidry band as well as the antifog properties of the insulators in polluted areas. The texture consists of a regular geometrical pattern providing the insulators with several advantages, such as a reduction of the leakage current in the shank part because of the larger surface area [1719]. Waters et al. [18] have studied the erosion and tracking of different textured SIRs in a series of inclined-plane tests. Contiguous hexagonal pattern, hexagonal intersections of overlapping protuberance s, and intersecting square arrangements all passed the inclined-plane test successfully, but plane SIR surfaces failed. Haddad et al. [17] studied the effect of a noncontiguous hemisphere texture, which gives a 1.49 times larger surface are a for the surface tracking and erosion of SIR. Fog tests were also performed on the samples to study the performance of the textured SIR under polluted conditions. Lower tracking and erosion rates as well as smaller and more mobile partial arcs were observed on the textured SIR surface indicating better antifog properties [17]. Although textured SIR surfaces have shown better surface and electrical properties, the question of how the textured surface of the SIR might influenc e the degree of microbial adhesion and growth pattern has not been studied.
The aim of the present study was to investigate how the texture of the surface affects the degree of microbial growth on SIR by comparing contiguous part-spherical protuberance textured SIR and plane surface SIR. The surface wetting properties of the textured and plane samples were studied to assess the effect of texture on the hydrophobicity of the material. Microbial growth tests with fungi were performed in microenvironment chambers $[7,8]$ with samples hanging horizontally and vertically to represent different parts of the insulator housing. Samples were studied visually and photographed to evaluate the difference in growth patterns on the surfaces of the textured and plane SIRs. Image analysis methods were developed and applied to evaluate the intensity and the degree of growth.

\section{Materials and Methods}

2.1. Material and Sample Preparation. Sylgard 184 from Dow Corning, consisting of a base and a cross linker agent, was purchased from GA Lindberg, Sweden. Textured surface samples were prepared by mixing the base and curing agent in a ratio of $10: 1$ in accordance with the suppliers' instruction and the mixture was degassed for $30 \mathrm{~min}$ to remove air bubbles. To create a textured surface, samples were cured for $48 \mathrm{hrs}$ in an aluminum mould with dimensions of $10 \times 10 \times$ $0.2 \mathrm{~cm}^{3}$ producing a pattern of contiguous hemispherical protuberances $2.5 \mathrm{~mm}$ in diameter on one side, giving rise to 1.907 times higher surface area [17]. Figures 2(a) and 2(b) show the aluminum mould and Figure 2(c) shows the top view of the textured sample with an area of the $1 \times 1 \mathrm{~cm}^{2}$ (taken by a real-time camera equipped with a $55 \mathrm{~mm}$ focal length objective).

Plane surface SIR samples were prepared by mixing the base and the curing agent in a ratio of $10: 1$, degassing the mixer for $30 \mathrm{~min}$ and curing the mixture in a Teflon mould with dimensions of $2 \times 7 \times 0.2 \mathrm{~cm}^{3}$ for $48 \mathrm{hrs}$. Samples of the textured and plane SIR with dimension $2 \times 7 \times 0.2 \mathrm{~cm}^{3}$ were prepared and sterilized by exposure to UV radiation for $5 \mathrm{~min}$ on each side prior to the microbial growth tests.

\subsection{Microorganisms. Cladosporium cladosporioides (DSM} 19653), previously isolated from HV insulators in Sweden [5], was provided as an active culture from DSMZ, the German Resource Center for Biological Materials. The fungus was recultured on malt extract agar and incubated at room temperature. Fresh fungal cultures were recultured 14 days prior to the growth test and incubated at room temperature.

2.3. Microbial Suspension Preparation. A fungal spore suspension was prepared by pipetting $5 \mathrm{~mL}$ of sterile mineralsalt/wetting-agent solution, prepared according to ISO $846: 1997$, on the surface of the fungi culture. Spores were collected by scraping the wetted fungi surface and were filtered through glass wool. This procedure was repeated 3 times and the filtered spore suspension was thereafter centrifuged twice. Finally, the spores were suspended in the sterile mineral-salt/glucose solution prepared according to ISO $846: 1997$. The number of the spores was adjusted to $10^{6}$ spores per $\mathrm{mL}$. 


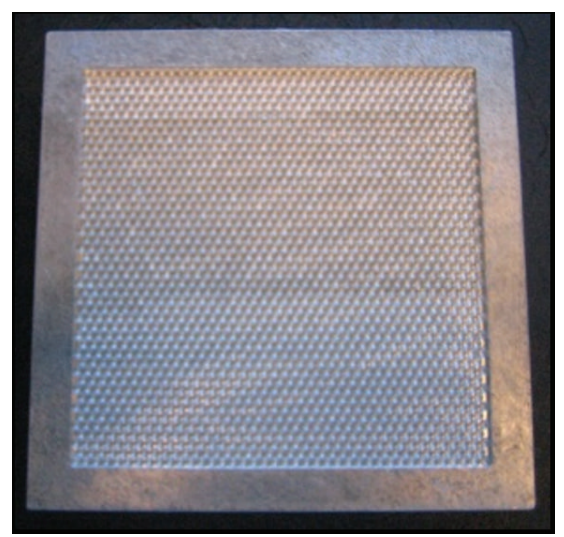

(a)

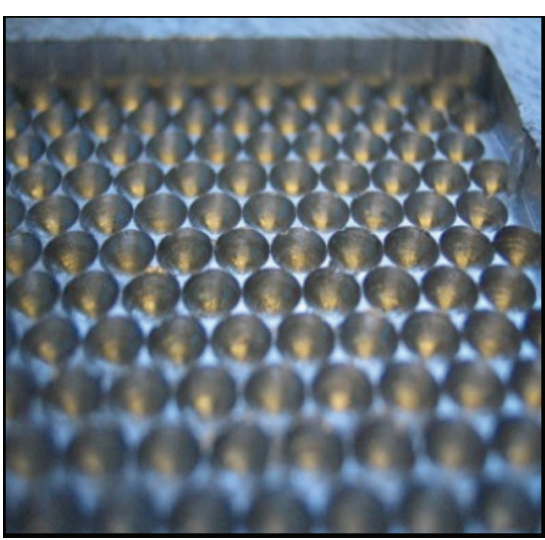

(b)

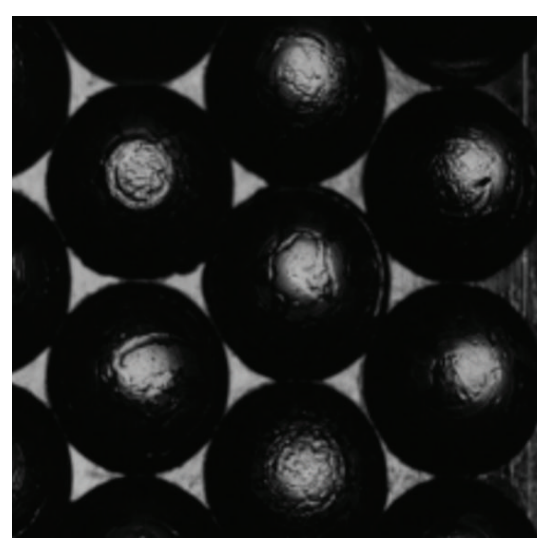

(c)

Figure 2: (a) The empty aluminum mould with dimension of $10 \times 10 \times 0.2 \mathrm{~cm}^{3}$. (b) A closer view of the empty aluminum mould. (c) Top view of the textured sample.

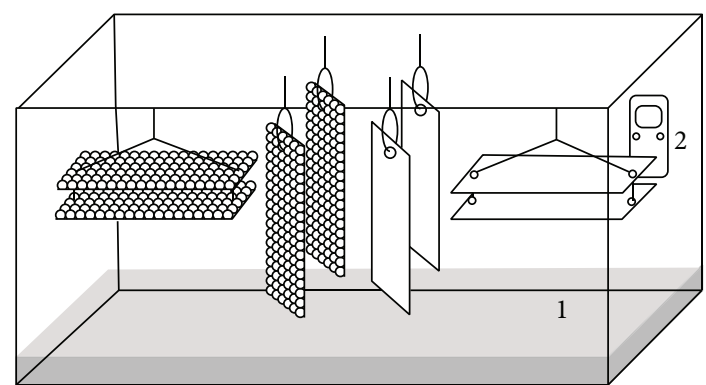

(1) Water about $5 \mathrm{~cm}$ deep

(2) Thermometer/hygrometer

Figure 3: Microenvironment chamber [7, 8].

2.4. Microenvironment Chamber Test. In the microenvironment chamber, two textured and two plane samples with dimensions of $2 \times 7 \times 0.2 \mathrm{~cm}^{3}$ were hung vertically to represent the housing material in the shank region of an insulator and the same number of samples was hung horizontally and slightly tilted $\left(20^{\circ}\right)$ to represent the housing material in the shed part of an insulator (Figure 3). A sterile atomizer was used to inoculate each sample with $7 \mathrm{~mL}$ of spore suspension. The chamber was placed at room temperature and the humidity was adjusted to $\geq 90 \%$. Fresh spore suspension was sprayed onto the samples once a week for four weeks $(28 \mathrm{~mL}$ in total). Thereafter, samples were photographed without cleaning. Clean samples of plane and textured surfaces were photographed as references.

2.5. Contact Angle Measurements. Contact angles were determined using a Wilhelmy balance [20] from Krüss K12. Samples were hung from a balance perpendicular to the surface of the water and the interaction force between the sample and the water was measured. The contact angle is calculated according to the following equation:

$$
\tau=\frac{F}{L}=\gamma_{\mathrm{LV}} \cos \theta=\gamma_{\mathrm{SV}}-\gamma_{\mathrm{SL}},
$$

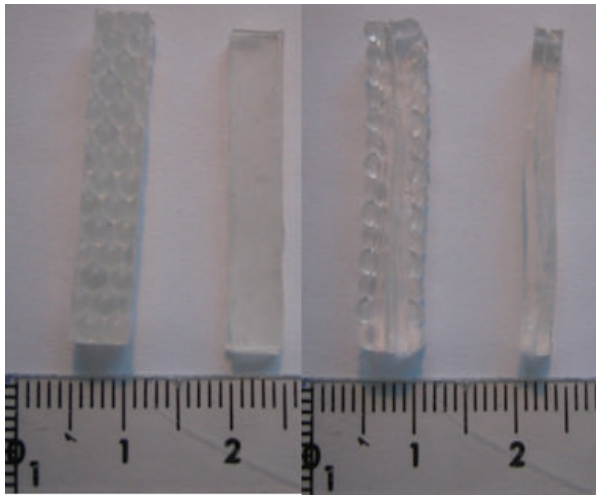

FIGURE 4: Samples prepared for contact angle measurements.

where $F$ is the interaction force between solid and liquid corrected for the liquid's buoyancy force, $L$ is length of the perimeter of the sample, $\theta$ is the contact angle between the water and the sample surface, $\gamma_{x y}$ is the interfacial tension between $x$ and $y$, and $\tau$ is the wetting tension.

To have the same texture on both surfaces, two pieces of the textured surface sample with the same dimension were cut and taped together, the total dimensions of the sample then being $5 \times 5.3 \times 30 \mathrm{~mm}^{3}(0.817 \mathrm{~g})$. The thickness of the sample was calculated considering the sample's density and weight. Two pieces of the plane surface SIRs with the same dimensions were also taped together giving the total dimensions of $5 \times 4 \times 30 \mathrm{~mm}^{3}(0.6626 \mathrm{~g})$. Samples are present in Figure 4.

Samples were cleaned with ethanol and MilliQ water and dried prior to the measurements. The surface tension of the pure water was determined to be $72 \pm 0.2 \mathrm{mN} \mathrm{m}^{-1}$ in a separate measurement. Two measurements were performed on each sample. In the first test, the sample was immersed in the water to a maximum depth of $10 \mathrm{~mm}$, withdrawn to a depth of about $1 \mathrm{~mm}$, and immersed in the water again to a depth of $10 \mathrm{~mm}$ and finally withdrawn to a depth of zero. In the second test, samples were immersed to a depth of $10 \mathrm{~mm}$ and 
withdrawn. The water was changed between the first and the second tests. The speed of the measurements was $2 \mathrm{~mm} \mathrm{~min}^{-1}$ and the temperature of the water was kept constant at $24^{\circ} \mathrm{C}$.

2.6. Image Analysis. The eight samples from the microenvironment chamber and the two reference samples were placed on a white luminescent surface and were simultaneously photographed from a distance of $20 \mathrm{~cm}$ in a dark room. High image contrast and few errors due to reflections and other photographic conditions were thus obtained. The resulting image was exported to MATLAB program for analysis. Each image was represented as a set of three matrices with intensity values of red, green, and blue components and as a grayscale image obtained by averaging over the three colors. Four different methods were developed and studied in order to analyze the area covered by microbial growth on each sample. Method 1 compared the gray-scale image intensity with a predefined threshold value chosen as 0.5 , and the microbial growth area was calculated as the fraction of pixels with higher intensity value than 0.5 . This value was chosen slightly too high to capture all the growth in order to avoid appearance of the anomalous effects of the textured samples. Method 2 was based on the observation that regions with microbial growth often exhibit a relatively large difference between the blue and red intensity values. A threshold of 0.1 was chosen, and the microbial growth area was calculated as the fraction of pixels with a red-blue intensity difference greater than 0.1. Method 3 is a combination of methods 1 and 2. The pixels that are filled by either of those methods are filled in method 3, thus typically giving a slightly larger growth area than either of the first two methods. It should be noted that the choice of threshold is dependent on the light conditions during photographing. Method 4 was based on the hypothesis that the threshold for microbial growth can be found by identifying the regions where the intensity starts to increase rapidly. The gray-scale intensity data was sorted in increasing order and plotted against the area fraction. The growth fraction was then determined as the leftmost position on the curve with a 45 degrees slope.

\section{Results and Discussion}

3.1. Comparison of the Hydrophobicity of Plane and Textured SIRs. The textured structure allows water to accumulate on the surface, promoting changes in the insulator's surface properties and accelerating biological growth. The hydrophobicity of the textured structures is therefore important. The hydrophobicity and wetting properties of the plane and textured samples were studied by determining the dynamic contact angle with a Wilhelmy balance [20]. Figure 5(a) shows contact angles of the plane SIR. SIR is a hydrophobic material and the advancing contact angle is higher than $90^{\circ}$ when the immersion in water begins. The contact angle increased to about $140^{\circ}$ as the sample was immersed deeper into the water. When the sample was withdrawn from the water, the contact angle decreased and the receding contact angle was about $40^{\circ}$ when the withdrawal was complete. These values for the advancing and receding contact angles were repeated during the second cycle indicating that prewetting of the sample did not affect the hydrophobicity. The curve shows that the contact angle hysteresis is time-dependent, and the longer the immersion time the higher the hysteresis. This is presumably due to a reorientation of the methyl groups on the surface of SIR [21]. The unusually large hysteresis can also be partially explained by the fact that the samples were cleaned prior to contact angle measurements, which resulted in the removal of silicone oligomers from the surface, and enhanced the interaction of the silica particles (fillers) and water.

The dynamic contact angles of the plane SIR were also evaluated using the sessile drop technique [15]. A $20 \mu \mathrm{L}$ drop of water was placed on the surface through a syringe and water was injected into the drop until the total volume reached $30 \mu \mathrm{L}$. The liquid was then immediately withdrawn and the advancing and receding contact angles were calculated. The advancing and receding contact angles were $125^{\circ}$ and $50^{\circ}$, respectively, which is in agreement with the data obtained using Wilhelmy balance.

Figure 5(b) shows results for the contact angle measurements of the textured samples. The advancing contact angle increased to about $130^{\circ}$ when the sample was immersed in water and the receding contact angle decreased to $60^{\circ}$ when the sample was withdrawn from the water. In this case, however, a stick-slip behavior was observed for both advancing and receding contact angles due to the structure of the surface. This sample has a lower contact angle hysteresis than the plane SIR. The second test contact angle results of both textured and plane samples are presented in Figure 5(c). The advancing contact angle behavior of the two samples was similar, but the textured sample had a higher receding contact angle than the plane sample. It can be concluded that the texture of the surface influences the dewetting properties of the sample and that it is easier for the water to be released from the surface of the textured sample.

Dry band arcing is initiated when local regions of a wet surface are dried due to high local leakage currents across the high resistive dry region because the wet regions on the insulator are more conductive. Pollution and moisture conditions combined together often increase the risk of surface leakage currents. The textured surface on an insulator spreads the water channels homogenously over the surface since the water needs to pass through the regular structure on the surface, and the texture reduces the risk of overheating of a particular area on the insulator because the leakage current is uniformly distributed on the surface $[17,18]$. The larger receding contact angle, that is, less hysteresis, therefore suggests that the textured structure on the surface reduces the risk of insulator failure due to dry band formation.

3.2. Visual Examination and Image Analysis of Biological Growth. HV insulator materials have been extensively studied with regard to determination and prevention of microbial growth [4-15]. It is important to examine how changes in surface morphology affect the biofilm formation which is inevitable during the lifetime of an insulator. To compare the textured materials with the conventional plane surface material, samples were inoculated with fungi in the microenvironment chamber test, Figures 6(a) and 6(b). 


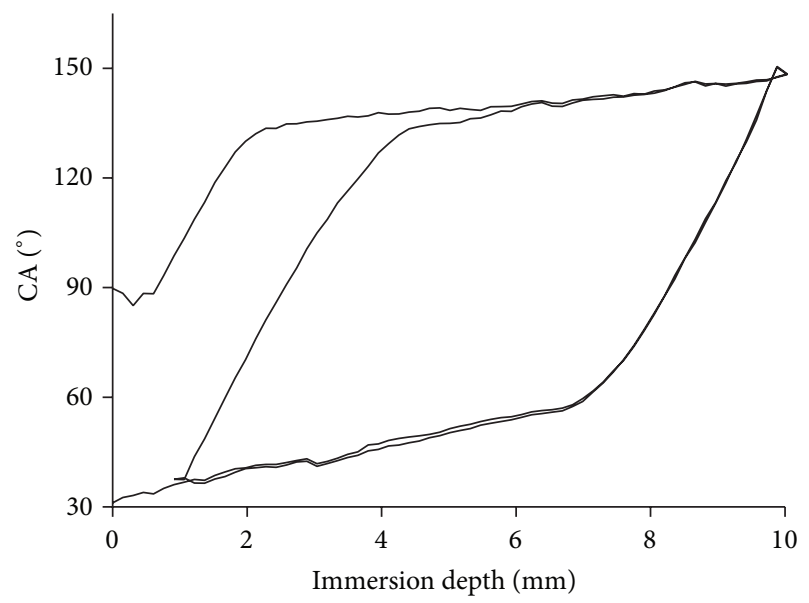

(a)

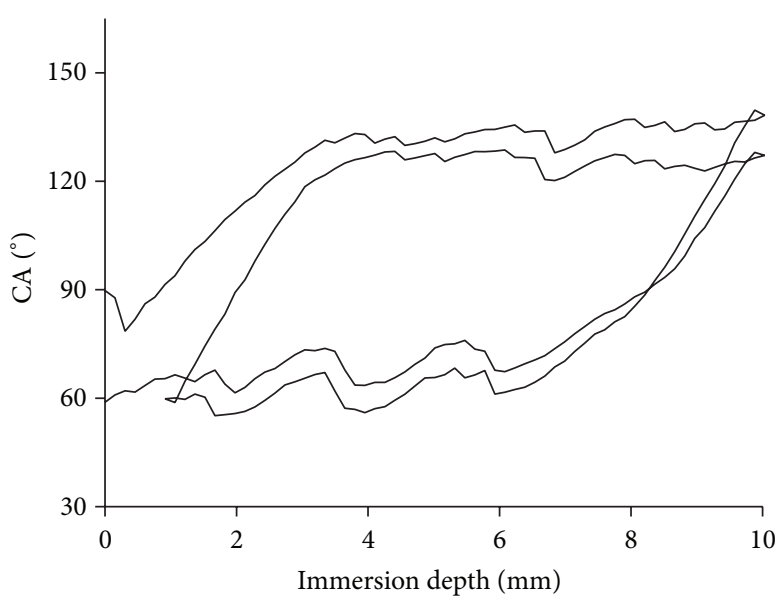

(b)

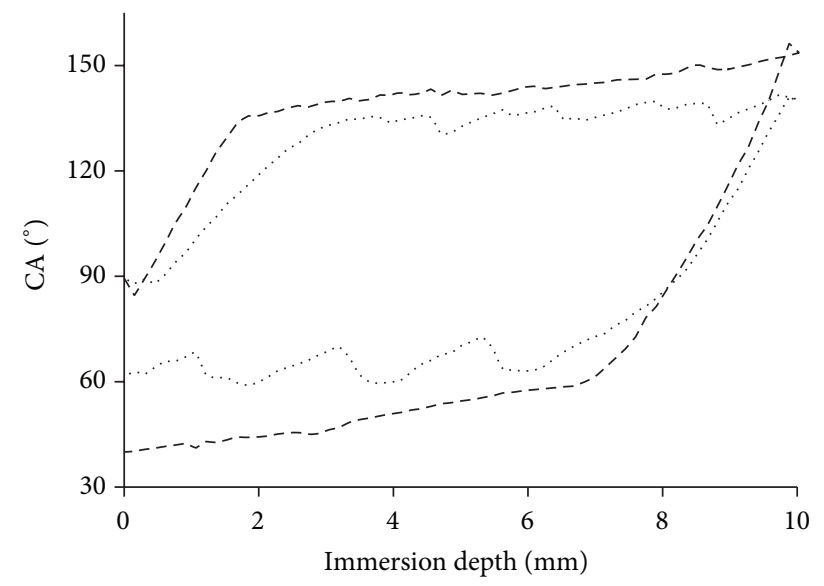

(c)

FIGURE 5: Dynamic contact angles versus immersion depth (a) plane surface SIR, test 1; (b) textured surface SIR, test 1; (c) samples in test 2 (- -) plane surface and (...) textured surface.

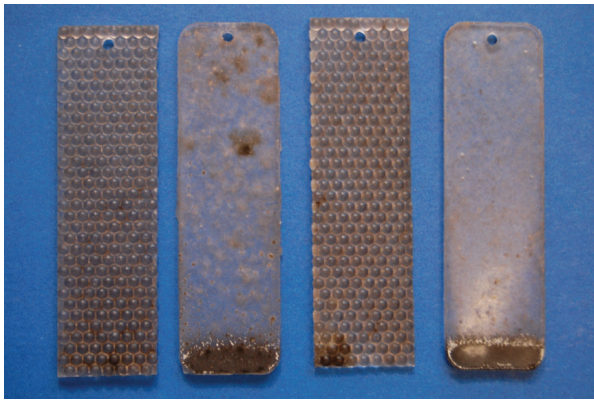

(a)

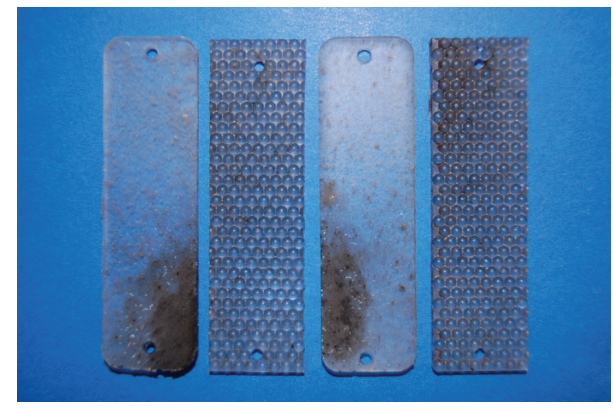

(b)

FiguRE 6: Samples removed from the microenvironment chambers after 28 days. (a) Vertically hung samples and (b) horizontally hung samples.

The fungal growth was most intense in a region close to the lowermost edge of the vertically hung plane samples and was concentrated to a slightly larger region on the downwards tilted side of the horizontally hung plane samples. The growth was more evenly distributed on the surface of both the vertically and the horizontally hung textured samples, with higher concentrations in the gaps between the regular structures with almost no growth on top of the structures. Since visual examination gives only an overview of how the fungal growth was distributed on the materials, image analysis was performed to give more precise information with a possibility to quantify the biofilm formation on the 
TABLE 1: Results from the image analysis: area covered with microbial growth (\%).

\begin{tabular}{|c|c|c|c|c|}
\hline \multirow{2}{*}{ Samples } & \multicolumn{4}{|c|}{ Methods } \\
\hline & Method 1 & Method 2 & Method 3 & Method 4 \\
\hline Plane surface horizontally hung & $7.85 \pm 0.52$ & $8.66 \pm 1.75$ & $13.15 \pm 1.97$ & $15.79 \pm 0.44$ \\
\hline Plane surface vertically hung & $12.45 \pm 1.76$ & $19.83 \pm 3.02$ & $24.50 \pm 0.09$ & $28.84 \pm 3.97$ \\
\hline Textured surface horizontally hung & $13.30 \pm 1.62$ & $15.91 \pm 13.33$ & $22.48 \pm 9.87$ & $9.90 \pm 0.7$ \\
\hline Textured surface vertically hung & $10.00 \pm 1.32$ & $5.48 \pm 4.06$ & $12.71 \pm 3.45$ & $12.46 \pm 2.44$ \\
\hline Reference plane surface & 0.02 & 0.26 & 0.28 & 0.26 \\
\hline Reference textured surface & 0.99 & 0.53 & 1.5 & 3.2 \\
\hline
\end{tabular}
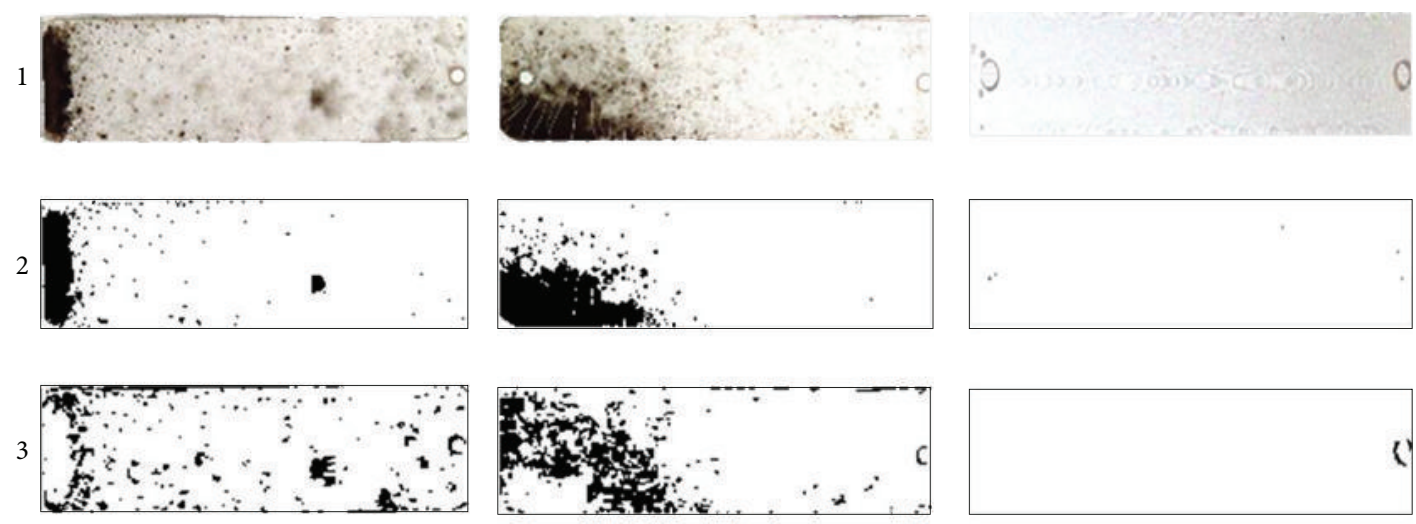

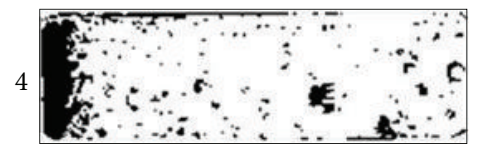

(a)

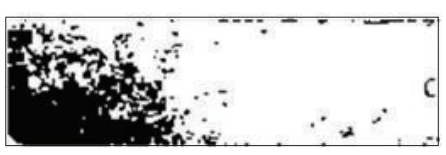

(b)

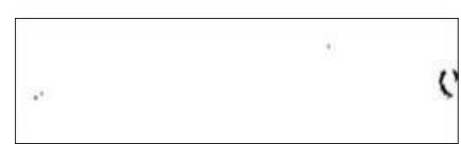

(c)

FIGURE 7: Image analysis of plane surface samples. (a) Vertically and (b) horizontally positioned samples and (c) reference sample. Row 1-4: original picture, images processed with method 1 , method 2 , and method 3.

surfaces. In order to describe the dens microbial growth, a threshold was used to eliminate the influence of the slight growth or dead microorganisms deposited on the surface. The percentage areas with dense microbial growth calculated with four image analysis techniques are summarized in Table 1. Each value is the average value for two samples ( \pm are the deviations). For the reference samples, only one sample was used in each case.

All methods give values in the range of $0.02-0.28 \%$ for the plane reference sample, which is very close to the correct value of $0.0 \%$. For the textured reference sample, all the methods give slightly larger values $(0.53-3.2 \%)$. The textured reference sample is more sensitive to the choice of threshold than the plane reference sample, especially in the case of method 1 . The underlying reason is that the texture itself causes both intensity and color differences. However, a small decrease in the threshold using method 1 can result in large errors for analysis of microbial growth on the textured sample. Method 1 allows determination of the sporulating microbial growth but is less sensitive to the less pronounced growth, which is captured by method 2 . However, method 2 is rather unstable on its own since it describes completely black regions as being without organic growth and gives rather large deviations for the textured surfaces. Therefore, the combination of the two methods, that is, method 3 , gives the most reliable results, although the deviation is highly affected by the limitations of method 2 .

The results from image analysis showed that for the plane samples all methods indicated more growth on the vertically hung samples than on the horizontally positioned samples. The vertically hung samples had a smaller but more intensive region covered with microbial growth, in accordance with visual examination. However, for the textured samples, values obtained from methods 1-3 are higher for the horizontally hung samples than the values obtained for the vertically hung samples. In contrast to the other methods, method 4 shows more growth on vertical textured samples. One possible explanation is that since the regular structure on the surface forces the growth to remain relatively evenly spread over the textured surfaces the concentration only becomes sufficiently high for exceeding the thresholds in the vertical cases. Figures 7 and 8 visualize how the different methods estimate the growth on plane and textured samples. Figures 7 (a) and 8 (a) present images for the samples hung vertically, Figures $7(\mathrm{~b})$ and 8 (b) show the samples hung horizontally, and Figures 7(c) and 8(c) present the reference samples. 

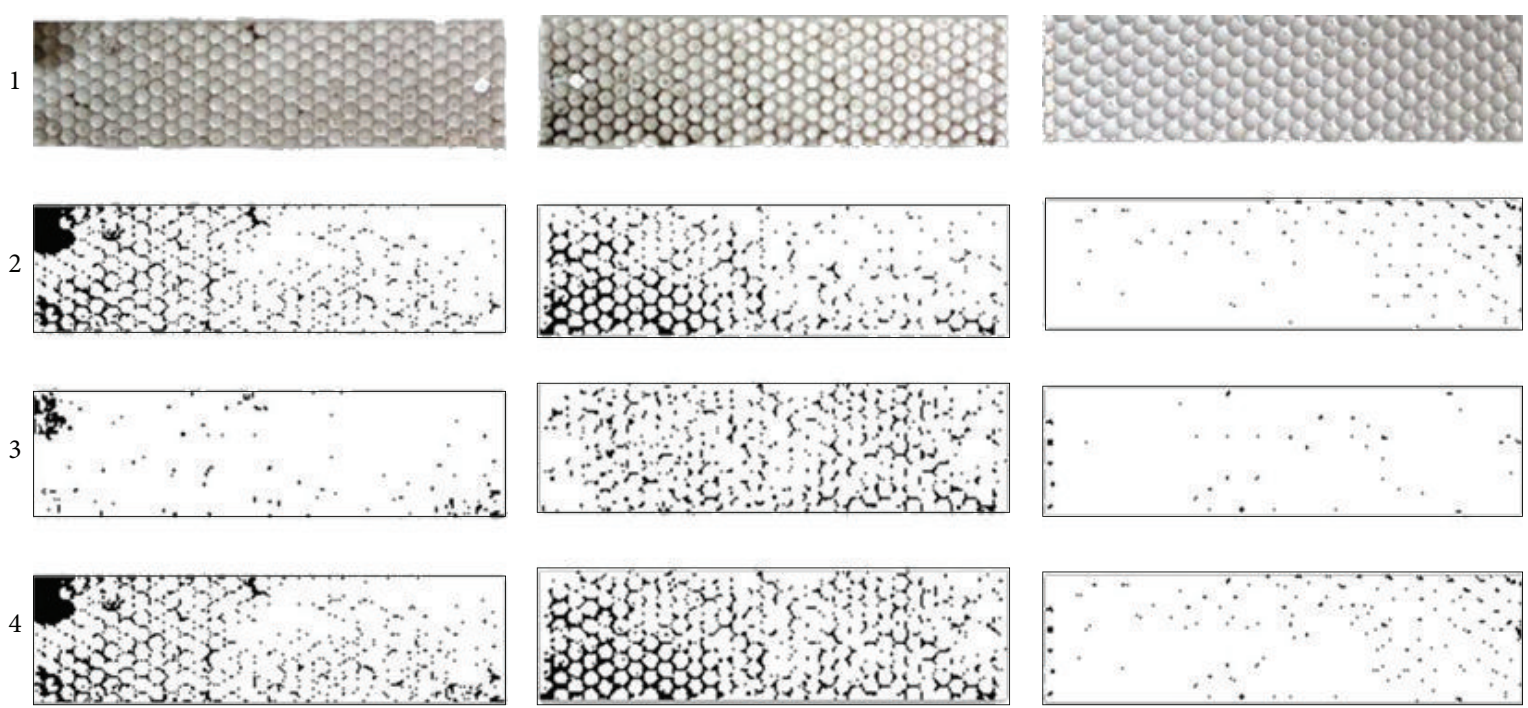

(a)

(b)

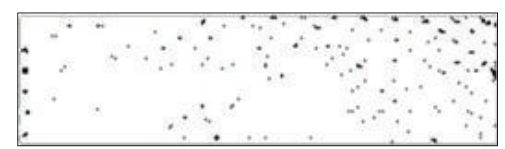

(c)

FIGURE 8: Image analysis of textured surface samples. (a) Vertically and (b) horizontally positioned samples and (c) reference sample. Row 1-4: original picture, image processed with method 1 , method 2 , and method 3.

The first row shows the original photos prior to analysis, the second row is the images processed by method 1 , the third is the images processed with method 2, and the fourth row shows the images processed with method 3. Method 4 operates on sorted data and can thus not be visualized in this way. Method 3 clearly gives the most realistic coverage of these three methods while method 2 is least reliable, as discussed above. Method 1 with a lower threshold can however be a sufficiently good alternative if only plane samples are to be analyzed.

The analysis from method 3 provides detailed information on how the biofilm forms on the surfaces of the samples. For the plane samples hung vertically the fluid flowed down the surface easily and was concentrated on the lower edges of the samples. This could also be observed for the tilted horizontally hung samples, where the fluid traveled towards the lower tilted side. However, dropwise growth was also present on both vertically and horizontally placed samples due to surface wetting properties of SIR.

Also for the textured samples hung vertically, the spore suspension traveled to the lower part of the surface and some intensive growth area was observed on the lower edges of the samples. However, the growth area for these samples was not as large as for the plane samples since some retention of the spores was observed between the regular structures throughout the sample surface, allowing more evenly dispersed microbial growth. For the horizontally hung textured samples, the area covered by microorganisms was almost doubled compared to the vertical samples, according to image analysis, method 3. The microbial growth was present almost everywhere on the surface of the horizontal samples and was more intensive on the tilted edges, similar to the plane surfaces. The suspension traveled in a different pattern for the samples hung horizontally compared to the samples hung vertically. It seems that the fluid has easier access to the gaps between the regular structures on the surface for the horizontal samples and microbial growth covered larger area on the surface of these samples than for the samples hung vertically. These results are in correlation to the results from the contact angle measurements, and the water is more evenly distributed over the material surface of the textured samples, allowing microorganisms to access a larger area of the sample.

From the visual and image analysis it can be concluded that the coverage of microbial growth highly depends on which part of the insulator is considered for the specific surface structure, that is, if the surface is going to be used as shank or shed parts of the insulators. The microbial growth is directly dependent on the water accumulation on the surface. The textured surfaces promote an even distribution of water, especially between the regular structures, supporting a more homogeneous microbial growth. The overall tendency is that the growth becomes more well distributed and slightly less pronounced with textured than plane samples and that vertical alignment gives a more intense growth but on a smaller area.

\section{Conclusions}

The textured SIRs lower the risk for dry band formation due to equal distribution of the water on the surface, therefore improving the electrical properties of the silicone rubber insulation material. Higher receding contact angle and subsequent lower hysteresis were observed due to the texture on the surface. The use of the textured surface should, however, be restricted to vertical areas of the insulator (shank), since use of the novel structured surfaces as the sheds in housing of the insulator might promote biofilm formation and consequently influence the electrical properties of the application. The water distribution on the surface of horizontally placed materials supported higher distribution of the microbial growth, 
ultimately changing the surface properties of the resulting material. Since biofilm contains vast amounts of incorporated water, it can be concluded that the surfaces of the insulators will be highly affected. The visual examination of the samples gives only a rough estimation of the distribution of the microbial growth on the surface. It is therefore essential to use the image analysis methods to determine the precise amount and pattern of growth for each specific surface and exposure. The image analysis showed that the microbial growth on the plane surface samples hung vertically was more pronounced compared to the samples hung horizontally; the growth was, however, more intensive in limited areas. The results from the analysis of the textured surfaces showed a more homogeneous distribution of microorganisms on the surface of both vertically and horizontally hung samples, with some accumulation of the microorganisms in the lower part of the vertically hung sample. This accumulation was not as pronounced as for the plane surfaces. The results also showed that the amount of microorganisms on the surface of horizontally hung textured samples was almost doubled compared to the plane surfaces.

\section{Conflict of Interests}

The authors declare that there is no conflict of interests regarding the publication of this paper.

\section{Acknowledgments}

Financial support given by Energimyndigheten, Elforsk, and ABB Corporate Research Center through the Elektra program is gratefully acknowledged.

\section{References}

[1] E. A. Cherney, "Non-ceramic insulators-a simple design that requires careful analysis," IEEE Electrical Insulation Magazine, vol. 12, no. 3, pp. 7-15, 1996.

[2] R. Hackam, "Outdoor HV composite polymeric insulators," IEEE Transactions on Dielectrics and Electrical Insulation, vol. 6, no. 5, pp. 557-585, 1999.

[3] H. Hillborg, M. Sandelin, and U. W. Gedde, "Hydrophobic recovery of polydimethylsiloxane after exposure to partial discharges as a function of crosslink density," Polymer, vol. 42, no. 17, pp. 7349-7362, 2001.

[4] S. Kumagai, "Influence of algal fouling on hydrophobicity and leakage current on silicone rubber," IEEE Transactions on Dielectrics and Electrical Insulation, vol. 14, no. 5, pp. 1201-1206, 2007.

[5] S. Wallström, K. Dowling, and S. Karlsson, "Development and comparison of test methods for evaluating formation of biofilms on silicones," Polymer Degradation and Stability, vol. 78, no. 2, pp. 257-262, 2002.

[6] S. Wallström and S. Karlsson, "Biofilms on silicone rubber insulators; microbial composition and diagnostics of removal by use of ESEM/EDS - composition of biofilms infecting silicone rubber insulators," Polymer Degradation and Stability, vol. 85, no. 2, pp. 841-846, 2004.
[7] S. Wallström, E. Strömberg, and S. Karlsson, "Microbiological growth testing of polymeric materials: an evaluation of new methods," Polymer Testing, vol. 24, no. 5, pp. 557-563, 2005.

[8] S. Wallström, A. D. Dernfalk, M. Bengtsson, S. Kröll, S. M. Gubanski, and S. Karlsson, "Image analysis and laser induced fluorescence combined to determine biological growth on silicone rubber insulators," Polymer Degradation and Stability, vol. 88, no. 3, pp. 394-400, 2005.

[9] R. S. Gorur, J. Montesinos, R. Roberson, J. Burnham, and R. Hill, "Mold growth on nonceramic insulators and its impact on electrical performance," IEEE Transactions on Power Delivery, vol. 18, no. 2, pp. 559-563, 2003.

[10] F. Coowar and P. R. P. Hoole, "Discharge characteristics of algacontaminated string insulators in the tropics," Electric Power Systems Research, vol. 15, no. 3, pp. 215-218, 1988.

[11] R. D. McAfee, R. D. Heaton, J. M. King, and A. U. Falster, "A study of biological contaminants on high voltage porcelain insulators, I," Electric Power Systems Research, vol. 42, no. 1, pp. 35-39, 1997.

[12] K. Naito, R. Matsuoka, and K. Sakanishi, "Investigation of the insulation performance of the insulator covered with Lichen," IEEE Transactions on Power Delivery, vol. 5, no. 3, pp. 1634$1640,1990$.

[13] M. Bengtsson, R. Grönlund, M. Sjöholm et al., "Fluorescence lidar imaging of fungal growth on high-voltage outdoor composite insulators," Optics and Lasers in Engineering, vol. 43, no. 6, pp. 624-632, 2005.

[14] M. Bengtsson, S. Wallström, M. Sjöholm et al., "Fungus covered insulator materials studied with laser-induced fluorescence and principal component analysis," Applied Spectroscopy, vol. 59, no. 8, pp. 1037-1041, 2005.

[15] S. Atarijabarzadeh, E. Strömberg, and S. Karlsson, "Inhibition of biofilm formation on silicone rubber samples using various antimicrobial agents," International Biodeterioration \& Biodegradation, vol. 65, no. 8, pp. 1111-1118, 2011.

[16] A. H. El-Hag, S. H. Jayaram, and E. A. Cherney, "Calculation of leakage current density of silicone rubber insulators under accelerated aging conditions," Journal of Electrostatics, vol. 67, no. 1, pp. 48-53, 2009.

[17] A. Haddad, R. T. Waters, H. Griffiths et al., "A new approach to anti-fog design for polymeric insulators," IEEE Transactions on Dielectrics and Electrical Insulation, vol. 17, no. 2, pp. 343-350, 2010.

[18] R. T. Waters, A. Haddad, H. Griffiths, N. Harid, P. Charalampidis, and P. Sarkar, "Dry-band discharges on polluted silicone rubber insulation: control and characterization," IEEE Transactions on Dielectrics and Electrical Insulation, vol. 18, no. 6, pp. 1995-2003, 2011.

[19] R. T. Waters and A. Haddad, "Insulating structures," UK Patent 2406225 A, 2005.

[20] H. Hillborg, J. F. Ankner, U. W. Gedde, G. D. Smith, H. K. Yasuda, and K. Wikström, "Crosslinked polydimethylsiloxane exposed to oxygen plasma studied by neutron reflectometry and other surface specific techniques," Polymer, vol. 41, no. 18, pp. 6851-6863, 2000.

[21] M. J. Owen, "Siloxane surface activity," in Silicon-Based Polymer Science, vol. 224 of Advances in Chemistry, pp. 705-739, 1990. 

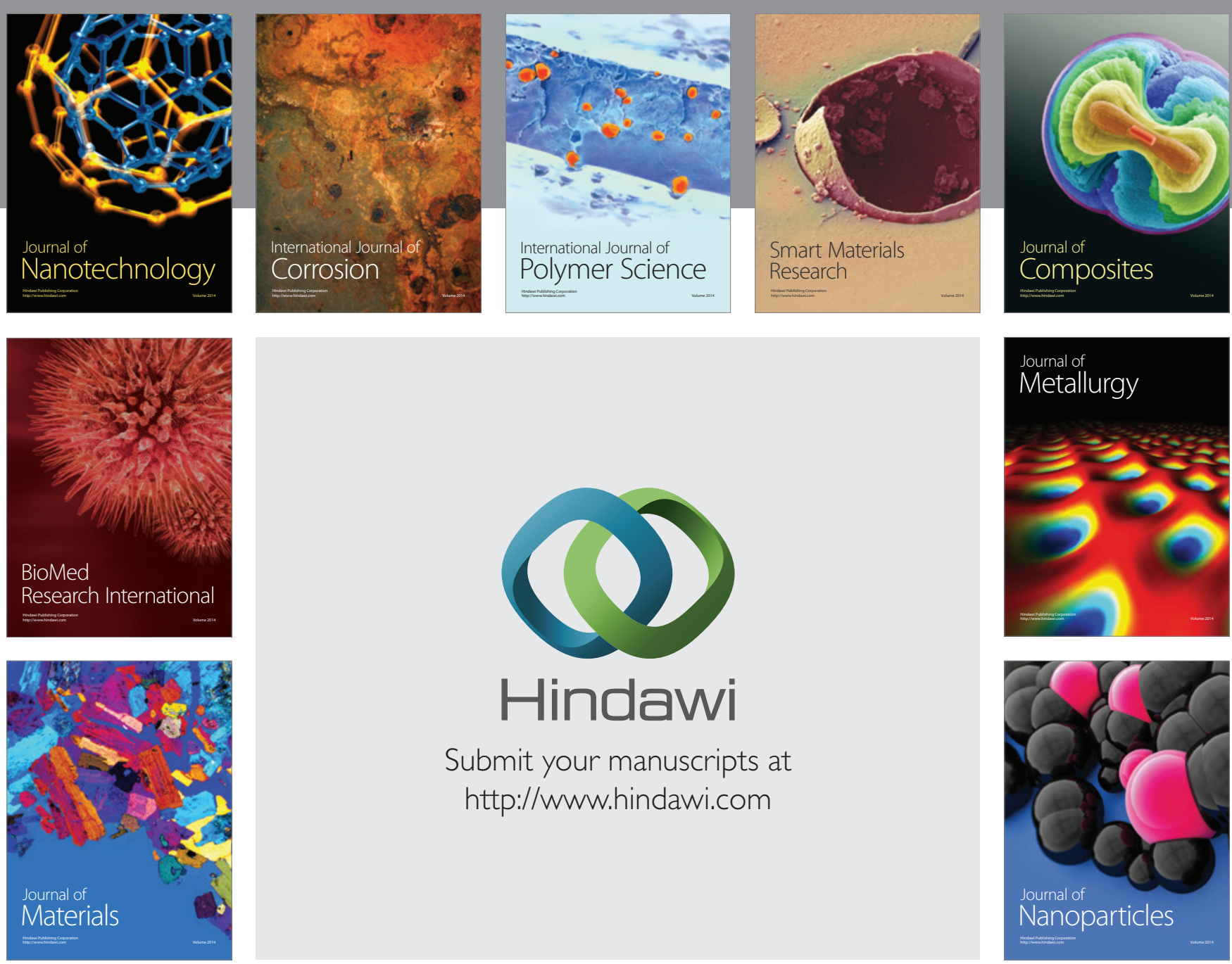

Submit your manuscripts at http://www.hindawi.com
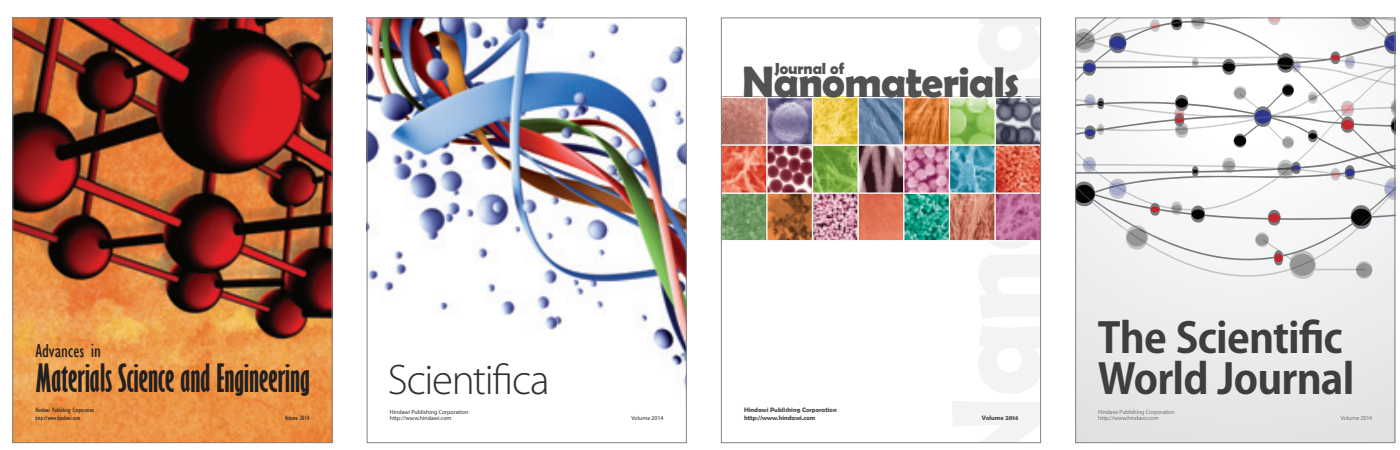

\section{The Scientific World Journal}
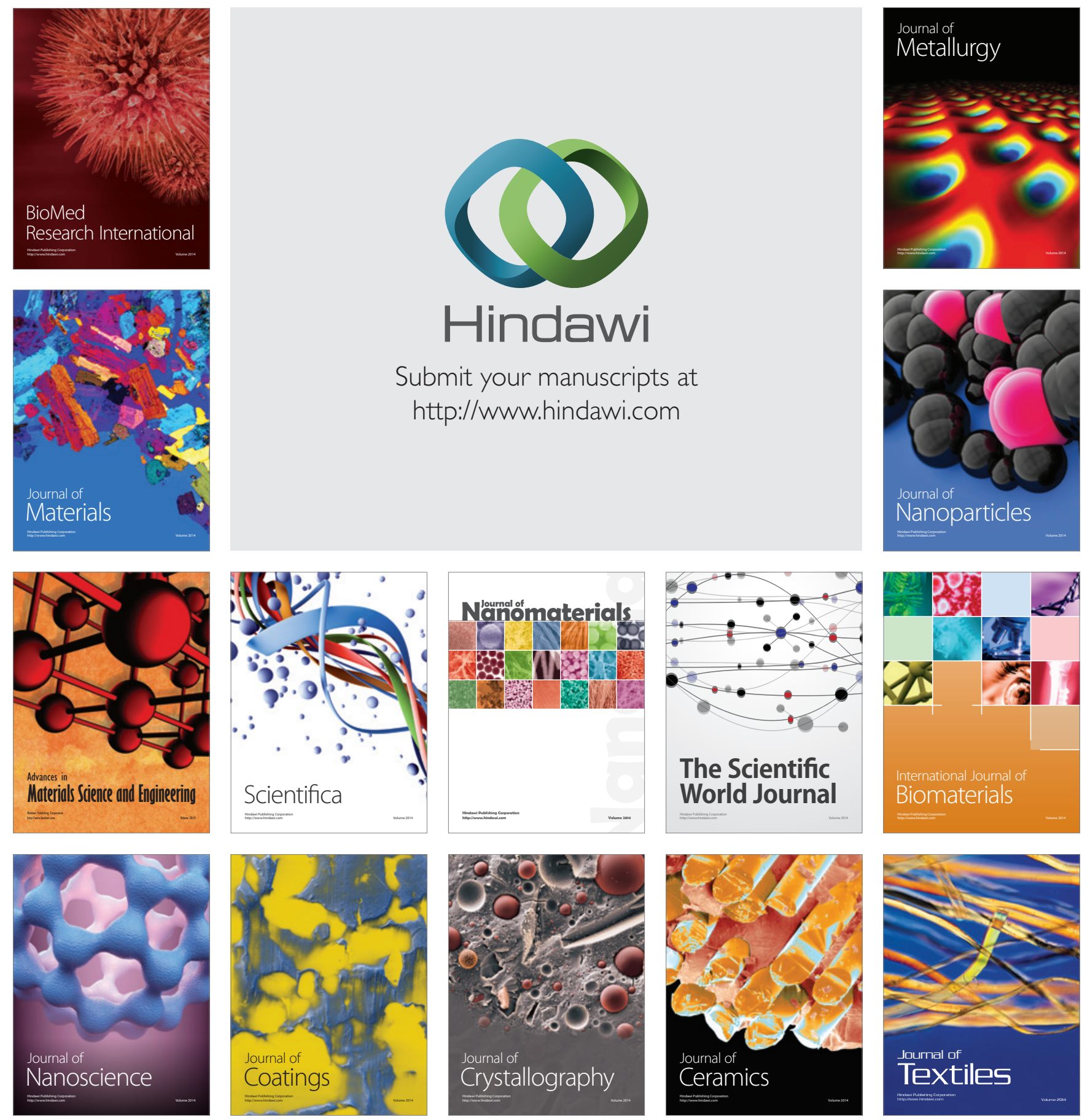